\title{
Doppelmoral hält besser: Die Politik mit der Solidarität in der Externalisierungsgesellschaft
}

\author{
Stephan Lessenich
}

Online publiziert: 10 . September 2020

(C) Der/die Autor(en) 2020

Zusammenfassung In der wissenschaftlichen Selbstbeschreibung des europäischen Wohlfahrtsstaats ist eine Erzählung dominant, die ihn als - wenn auch national unterschiedlich ausgeprägte - weltweit einzigartige Form der Institutionalisierung sozialer Solidarität versteht, die seit den 1980er-Jahren durch die neoliberale Transformation (gleichsam von außen) in Gefahr geraten sei. Der Beitrag setzt dieser Erzählung eine andere entgegen: Auch schon vor der ,neoliberalen Revolution“ lebte das europäische Solidaritätsarrangement davon, dass es auf Kosten eines gesellschaftlichen „Außen“ organisiert wurde und systematisch Dritte schädigte. Solidarität in den europäischen „Externalisierungsgesellschaften“ funktioniert nur als exkludierende Solidarität: Sie ist durch strukturelle soziale Schließungen nach innen, insbesondere aber - vermittelt über die Institution der ,citizenship“ - nach außen charakterisiert. Insofern zeichnet sich der europäische Wohlfahrtsstaat durch eine fundamentale normative Selbstüberschätzung aus: Indem er „Solidarität“ eindimensional als nationalgesellschaftlichen Zusammenhalt ausbuchstabiert, verfährt er tatsächlich nach einem doppelten moralischen Standard.

Schlüsselwörter Solidarität · Wohlfahrtsstaat · Basiserzählung · Neoliberale Revolution · Externalisierung $\cdot$ Externalisierungsgesellschaft

\footnotetext{
S. Lessenich $(\bowtie)$

Institut für Soziologie, Ludwig-Maximilians-Universität München, Konradstr. 6, 80801 München, Deutschland

E-Mail: stephan.lessenich@soziologie.uni-muenchen.de
} 


\title{
Doubling down on double standards: The politics of solidarity in the Externalization Society
}

\begin{abstract}
The European welfare state's predominant scientific self-description understands it, some national variation notwithstanding, as a globally unique form of institutionalized solidarity that has come under attack from neoliberalism (from the outside, as it were) since the 1980s. This contribution counters this narrative with a different one: Even before the "neoliberal revolution" was the European arrangement of solidarity organized at the expense of a societal "outside" and inflicted systematic harm on third parties. Within the European "externalization societies", solidarity can function solely as exclusive solidarity: being characterized by structural social closure on the inside, though even more so - mediated by the institution of "citizenship" - on the outside. The European welfare state thus suffers from severe normative overconfidence: by unidimensionally treating solidarity as national cohesion, it indeed proceeds by a moral double standard.
\end{abstract}

Keywords Solidarity - Welfare state Essential narrative - Neoliberal revolution Externalization · Externalization Society

\section{La morale à géométrie variable de la politique de la solidarité dans la société externalisatrice}

Résumé La description scientifique de l'État-providence européen est dominée par un récit le présentant comme une forme d'institutionnalisation de la solidarité sociale certes variable d'un pays à l'autre mais globalement unique au monde qui serait mise en péril depuis les années 1980, en quelque sorte de l'extérieur, par la transformation néolibérale. À ce récit cet article entend opposer un autre récit: Même avant la « révolution néolibérale », le dispositif de solidarité européen était organisé aux dépens d'un «extérieur » social et systématiquement préjudiciable à des tiers. Dans les « sociétés externalisatrices » européennes, la solidarité ne fonctionne que comme solidarité exclusive: Elle est caractérisée par des clôtures sociales structurelles vers l'intérieur, mais aussi et surtout - par le biais de l'institution de la citoyenneté - vers l'extérieur. Aussi l'État-providence européen se caractérise-til par une surestimation de soi fondamentale en termes normatifs : En déclinant la « solidarité » de manière unidimensionnelle comme cohésion nationale, il procède de normes morales à géométrie variable.

Mots-clés Solidarité · État-providence · Récit premier · Révolution néolibérale · Externalisation $\cdot$ Sociétés externalisatrices

\section{Einleitung}

Zur Geschichte, Eigenart und Krise des Wohlfahrtsstaats hat sich in den Sozialwissenschaften und, über diese vermittelt, in der politischen Öffentlichkeit der Nachkriegsgesellschaften Westeuropas ein basaler Wissenskonsens etabliert. Ausgangs- 
punkt des folgenden Beitrags ist die Behauptung, dass sich dieser gesellschaftspolitische „,common sense“, dem der europäische Wohlfahrtsstaat als Arrangement institutionalisierter Solidarität gilt, aus einer in spezifischer Weise einseitigen wissenschaftlich-politischen Erzählung speist. Die Quintessenz meiner Überlegungen lautet, dass an dieser gleichsam offiziösen Erzählung alles richtig ist - und zugleich doch auch alles falsch. Sie steht in ihrer Doppelbödigkeit repräsentativ für eine hier näher zu bestimmende Politik mit der Solidarität, die sich keineswegs im Diskursiven erschöpft, sondern in vielfältiger Weise materiale Folgen zeitigt: in Gestalt nämlich von Strukturen sozialer Schließung, die sich in ihrer historischen Dynamik als bemerkenswert stabil erwiesen haben, in der Selbstbeschreibung des europäischen Wohlfahrtsstaates aber auffällig ausgeblendet geblieben sind. Da der Wohlfahrtsstaat europäischer Prägung zugleich aber - als Solidaritätsarrangement von seinen Befürworter- wie seinen Kritiker*innen immer auch stark moralisch aufgeladen wird, stellt sich wohlfahrtsstaatliche Politik mithin als ein prototypisches Beispiel jener gesellschaftlichen Doppelmoral dar, die als kulturelle Signatur der von mir so genannten Externalisierungsgesellschaft gelten kann.

\section{Alles richtig - und alles falsch: Die wohlfahrtsstaatliche Basiserzählung}

Unter einer Basiserzählung verstehe ich mit Thomas A. Herz die narrative Konstruktion bzw. Rekonstruktion eines historischen Sachverhalts, die die gesellschaftlich herrschende und somit als legitim anerkannte Version desselben vermittelt (vgl. Herz 1996). Als solche sind entsprechende Erzählungen grundsätzlich sozial umkämpft und unvermeidlicher Bezugspunkt allfälliger vergangenheitspolitischer Konflikte um die Deutung gesellschaftshistorischer Phänomene und Entitäten. Während Herz in seinen Studien den Nationalsozialismus und dessen nachfolgende Aufarbeitung als Basiserzählung der Bundesrepublik im Blick hatte, interessiert an dieser Stelle die wohlfahrtsstaatliche Basiserzählung vom sozialpolitisch organisierten gesellschaftlichen Zusammenhalt der deutschen und europäischen Nachkriegszeit gewissermaßen also die zivilisatorische Gegen- oder Komplementärerzählung zu jenem Narrativ, das seit den 1950er-Jahren die deutsche wie europäische Urerfahrung des faschistischen Zivilisationsbruchs gesellschaftlich handhabbar zu machen suchte.

In der einschlägigen sozialwissenschaftlichen Forschung ist besagtes Grundverständnis des eigenen Gegenstandes seit den 1980er-Jahren, gewissermaßen zeitgleich mit dem Höhe- und Wendepunkt des sozialpolitischen Nachkriegszyklus, als ,,sozialdemokratisches Modell“" wohlfahrtsstaatlicher Entwicklung bekannt - und dominant - geworden (vgl. Shalev 1983; Lessenich 2012, S. 73 ff.). Die ,story line“ der sozialdemokratischen Erzählung geht, in knapper, jedoch nicht unzulässig stilisierter Form dargeboten, ungefähr wie folgt:

Im Anfang war der industrielle Klassenkonflikt. Die historischen Wurzeln des modernen, demokratisch-kapitalistischen Wohlfahrtsstaats liegen demnach in der spezifischen Organisation gesellschaftlicher Produktion als von antagonistischen materiellen Interessen geprägtes Verhältnis von Lohnarbeit und Industriekapital. Seit 
Anbeginn des Industriekapitalismus virulent, kommt in die Konfliktdynamik dieser extrem asymmetrischen sozialen Beziehung - die Abhängigkeit der Lohnarbeitenden von den Kapitalbesitzenden ist ungleich existenzieller bzw. potenziell existenzgefährdender als umgekehrt (vgl. Offe und Wiesenthal 1985) - neue Bewegung mit der Demokratisierung der politischen Verhältnisse. Es ist die Umformung des betrieblichen Klassenkampfs in den „democratic class struggle“ (Korpi 1983), die der Gesellschaftsgeschichte ihre wohlfahrtsstaatliche Wendung gibt: Indem die Arbeiterklasse gegen die bis dato von Volkes Wille weitestgehend unbeschwert herrschenden Klassen das allgemeine und gleiche Wahlrecht erkämpft, stattet sie sich mit einer neuen, entscheidenden Machtressource aus, nämlich mit (zumindest indirektem) Einfluss auf die politische Gestaltung von industrieller Produktion, Arbeit und Beschäftigung. Der Wohlfahrtsstaat mit seinem stetig expandierenden System ,,arbeiterfreundlicher" Interventionen, vom betrieblichen Arbeits- und Gesundheitsschutz über den Kranz an Arbeitnehmerversicherungen und Familienleistungen bis hin zu einem frei zugänglichen öffentlichen Gesundheits- und Bildungswesen, ist dann der Institution gewordene Ausdruck des ,historischen Kompromisses“ zwischen Kapital und Arbeit: Zwar bleiben das Privateigentum an Produktionsmitteln und das betriebliche Direktionsrecht der Unternehmer im Kern unangetastet, doch nach dem regulativen Prinzip von,,politics against markets“ (Esping-Andersen 1985) wird die Marktabhängigkeit der Lohnabhängigen reduziert, werden ihre Arbeits- und Lebensverhältnisse zumindest ansatzweise dekommodifiziert, sprich vom stummen Zwang ihres Warencharakters befreit. Arbeit und Kapital begegnen sich im Wohlfahrtsstaat zwar immer noch nicht auf Augenhöhe, aber der industrielle Kapitalismus ist im späten 20. Jahrhundert, so das Zwischenfazit dieser Geschichte, zum demokratischen Kapitalismus domestiziert worden, er ist durch wohlfahrtsstaatliches Handeln zu einem Kapitalismus mit menschlichem Antlitz und sozialem Anspruch geworden.

Freilich haben sich im europäischen und transatlantischen Vergleich unterschiedliche Spielarten des demokratischen Wohlfahrtskapitalismus herausgebildet (vgl. Esping-Andersen 1990), die sich im Lichte der wohlfahrtsstaatlichen Basiserzählung als Variationen der Antwort auf die Frage darstellen, wie die Risiken der Lohnarbeiterexistenz (und, weitergehend, Lebenslaufs- und intergenerationale Risiken) vergesellschaftet werden. Die unterschiedlichen Wohlfahrtsstaatsmodelle lassen sich in diesem Sinne als verschiedenartige Solidaritätsmodelle beschreiben (vgl. EspingAndersen 1999, S. 40 ff.), als institutionelle Realisationsformen verschiedener - starker oder schwacher, ,dichter“ oder ,„dünner“ - Konzeptionen von Solidarität. In der sozialdemokratischen Erzählung sind diese Varianten zugleich mehr oder weniger explizit als normativ höher- oder minderwertig ausgewiesen (vgl. Manow 2002): Je stärker der Staat als Garant eines universalistischen, sämtliche gesellschaftliche Gruppen und Milieus, Schichten bzw. Klassen übergreifenden Risikoausgleichs in Erscheinung tritt, umso „besser“, sprich solidaritätspolitisch wertvoller. In dem Maße, in dem hingegen staatlicherseits Märkte und/oder Familienhaushalte als Instanzen sozialen Risikomanagements eingesetzt oder jedenfalls walten gelassen werden, wird die gesellschaftliche Solidaritätsluft dünner: Zwar können Risiken auch marktförmig gepoolt und reguliert werden, doch ist dann letztlich jeder einzelne Marktakteur auf sich selbst und seine bzw. ihre Eigenleistung angewiesen, um das je individuelle Wohlergehen zu sichern - mit dementsprechenden Ungleichheitskon- 
sequenzen. Und auch in einem intermediären Modell gemeinschaftlicher Solidarität, wo es mehr oder minder kleine Gruppen - von der Familie bis zum Berufsverband - sind, die sich im Falle des Risikoeintritts des wechselseitigen Bedarfsausgleichs (sei es nun im Sinne der Kinderbetreuung oder der Unfallhaftung) versichern, bleibt das Risikomanagement im Prinzip partikularistisch und ergeben sich entsprechend ungleiche Sicherungsniveaus und gruppenbezogen abgestufte Lebenschancen.

Soweit also die Erzählung vom Aufstieg des europäischen Wohlfahrtsstaats, die sich in der europäischen Sozialpolitikforschung weithin - auch über politisch der Sozialdemokratie zugeneigte wissenschaftliche Milieus hinaus - durchgesetzt hat: Aus dem industriellen Klassenkonflikt und den Demokratisierungskämpfen der Arbeiterbewegung hervorgegangen, war es wesentlich der jeweiligen Stärke derselben und ihren historischen Erfolgen oder Misserfolgen bei der Klassenkoalitionsbildung (vgl. Esping-Andersen 1990, S. 29 ff.) geschuldet, wie die nationalen Wohlfahrtsregime ausgestaltet wurden und ob sie das Solidaritätsprinzip stärker universalistisch, partikularistisch oder individualistisch ausbuchstabierten.

Wo Aufstieg war oder ist, kommt allerdings irgendwann auch einmal der Fall - so auch in der Geschichte des Wohlfahrtsstaats. Und damit setzt Teil zwei der Basiserzählung ein, der für weite Teile der sozialwissenschaftlichen Wohlfahrtsstaatsforschung prägend geworden ist. Wie bei der bundesrepublikanischen Basiserzählung, wo das Volk vom Nationalsozialismus gleichsam infiziert worden und dieser wie „die Pest über die Menschen von außen“ gekommen sein soll, ,als ein heimtückischer Überfall“ - so die retrospektive Deutung keines Geringeren als des ersten Nachkriegsvorsitzenden der Deutschen Gesellschaft für Soziologie, Leopold von Wiese (vgl. Dyk und Schauer 2015, S. $141 \mathrm{ff}$.), - kennt auch die wohlfahrtsstaatliche Basiserzählung das Bild der Überwältigung von außen. Fremde Mächte, die in diesem Fall durchweg als „Neoliberalismus“ firmieren, hätten demnach vom sozialen Kapitalismus Besitz ergriffen.

Schon in Teil eins der Erzählung waren es die Marktvergesellschaftung bzw. der liberale Wohlfahrtsstaat US-amerikanischer Spielart, die als institutioneller Gegenhorizont des europäischen Sozialmodells bzw. als das marktradikale Andere der Solidaritätsmodelle europäischer Prägung auftraten. Für die 1980er-Jahre weist das sozialpolitikwissenschaftliche Standardnarrativ dann dem ,angelsächsischen“ Wohlfahrtskapitalismus endgültig die Rolle als ,bad guy“ zu - als der böse Wolf, der die Solidarwelten jener europäischen Gesellschaften $\mathrm{zu}$ bedrohen begann, die sich doch erst wenige Jahrzehnte zuvor dazu durchgerungen hatten, ihre Bürger*innen mithilfe des sozialpolitischen Leviathans aus dem wohlfahrtsindividualistischen Naturzustand zu befreien. Die ,neoliberale“ Transformation des europäischen Wohlfahrtsstaats kam demnach von außen, von jenseits des Atlantiks oder zumindest des Ärmelkanals, aus den Häusern Reagan und Thatcher - und zerstörte das gute alteuropäische Modell des sozialpolitisch organisierten gesellschaftlichen Zusammenhalts.

Der Rest der Geschichte ist in diesem Sinne schnell erzählt: Die Kapitalseite fühlte sich durch den neoliberalen Gezeitenwechsel zur Änderungskündigung des demokratisch-kapitalistischen Nachkriegskompromisses politisch ermächtigt - und verfügte zudem über die entsprechenden Machtressourcen, um ihre Vorstellungen von der Gestalt eines neuen Akkumulationszyklus auch durchsetzen zu können (vgl. Streeck 2013, S. 34 ff.). Unter tätiger Mithilfe der europäischen Sozialdemokratie, 
die ihre Zukunft im Parteienwettbewerb allein über eine marktliberale Wende ihrer Programmatik und Politik gewährleistet sah (vgl. Nachtwey 2009), wurde die regulative Logik des europäischen Wohlfahrtsstaates konsequent auf ,politics for markets“ umgestellt. Der Neoliberalismus trat über sämtliche gesellschaftliche Lebensbereiche hinweg seinen Siegeszug an (vgl. Brown 2018), die schrittweise Rekommodifizierung der Arbeitskraft und die schleichende Vermarktlichung der Sozialpolitik führten zu fortschreitender gesellschaftlicher Entsolidarisierung und der Etablierung postwohlfahrtsstaatlicher Verhältnisse (vgl. Butterwegge 2015).

Was lässt sich zu dieser Erzählung, die von einer ihrem Gegenstand in häufig unverhohlener Sympathie verbundenen Sozialwissenschaft getragen wird, resümierend sagen? Wie eingangs angedeutet zweierlei. Zum einen ein schlichtes „Ja, so war es“: Man kann die Geschichte von Aufstieg und Fall des europäischen Wohlfahrtsstaates durchaus in dieser Weise rekonstruieren. Zum anderen ist damit allerdings nur die halbe Wahrheit gesagt. Oder anders: Diese Geschichte ist zu einfach - und einfach zu schaurig-schön, um wahr zu sein. Selbstverständlich gibt es denn auch heterodoxe Narrative, die freilich im wissenschaftlichen Wahrheitsspiel gegen den Mainstream der sozialdemokratischen Erzählung bis auf den heutigen Tag kaum durchzudringen vermögen.

Da wäre zuallererst die basale kapitalismustheoretische Gegenerzählung: Wohlfahrtsstaatliche Politik ging nie, zu keiner gesellschaftshistorischen Zeit, in ,,politics against markets“ auf, stets war sie zugleich auch marktermöglichend, immer - und in der demokratisch-kapitalistischen Gesellschaftsformation notwendigerweise immer - war sie gleichermaßen kommodifizierend wie dekommodifizierend (vgl. Lessenich 1999). Der demokratisch-kapitalistische Wohlfahrtsstaat ist ein von strukturellen Widersprüchen durchzogenes, ja selbstwidersprüchliches Arrangement (vgl. Borchert und Lessenich 2016, S. 48 ff.) - eine analytische Einsicht, die in der sozialdemokratischen Vergangenheitspolitik systematisch negiert und in gegenwartsbezogenen Neoliberalismusdiagnosen souverän unterlaufen wird (vgl. Lessenich 2017). Zwei weitere Gegenerzählungen dementieren ihrerseits, gleichsam im Zangengriff, die Rekonstruktion des europäischen Wohlfahrtsstaats als institutionalisiertes - und zumal als universalistisches - Solidaritätsarrangement. Die feministische Kritik am „sozialdemokratischen Modell“ zeigt schon seit den 1970er-Jahren auf, wie wohlfahrtsstaatliche Solidarität produktivitäts- und geschlechterpolitisch halbiert ist und die dem Kapital abgetrotzten sozialen Rechte im Kern solche des männlichen Normalarbeitnehmers sind (vgl. z.B. Orloff 1993). Die kritische Migrationsforschung wiederum weist auf darüber hinausgehende strukturelle Beschränkungen des wohlfahrtsstaatlichen Versprechens hin, insofern das Prinzip der ,citizenship rights“ immer auch eines der staatsbürgerlichen Exklusion und der aufenthaltsrechtlichen Stratifizierung von Rechten ist (vgl. z. B. Morris 2002) - wohlfahrtsstaatliche Solidarität ist, vor allen anderen sozialen Selektivitäten, zuallererst national strukturiert.

All diese analytischen Einwände gegen die wohlfahrtsstaatliche Basiserzählung nimmt das Konzept der „Externalisierungsgesellschaft“ auf - und problematisiert, gewissermaßen in der Kumulation von kapitalismus-, geschlechter- und migrationstheoretischer Kritik, auf ganz grundsätzliche Weise die wissenschaftliche Selbstbeschreibung des europäischen Wohlfahrtsstaats als Institutionalisierung gesellschaftlicher Solidaritätsbeziehungen. 


\section{Externalisierung: Die andere Seite des wohlfahrtsstaatlichen Solidaritätsarrangements}

Als „Externalisierung“ bezeichne ich einen spezifischen Vergesellschaftungsmodus, einen historisch situierten und räumlich umschriebenen Strukturmechanismus gesellschaftlicher Reproduktion. Kapitalistische Gesellschaften - oder, genauer, „Gesellschaften, in welchen kapitalistische Produktionsweise herrscht" (Marx 1986, S. 49) - sind Externalisierungsgesellschaften: Sie müssen zwangsläufig, ja geradezu zwanghaft externalisieren, um ihre Produktionsweise dauerhaft aufrechterhalten und auf stetig erweiterter Stufe reproduzieren zu können. Dieser für kapitalistische Gesellschaften konstitutive Reproduktionsmodus, der sich seit dem ausgehenden 16. Jahrhundert in den europäischen Zentren des kapitalistischen Weltsystems (vgl. Wallerstein 2004) etablieren konnte, begann sich auf eine zuvor unbekannte Weise zu verbreiten, zu vertiefen und zu verschärfen, als sich in eben jenen Zentrumsökonomien seit Mitte des 18. Jahrhunderts die industriekapitalistische Produktionsweise durchsetzte. Eingebettet in die historisch wechselnden politisch-ökonomischen Konfigurationen eines tendenziell global ausgreifenden Kapitalismus (vgl. Arrighi 1994), gewannen die Externalisierungsgesellschaften der westlichen Welt bzw. (so die spätere, umfassendere Bezeichnung) des globalen Nordens eine sozioökonomische, soziopolitische und soziokulturelle Entwicklungsdynamik, die sie im Verlauf des 20. Jahrhunderts unangefochten an die Spitze der weltgesellschaftlichen Sozialstruktur aufsteigen ließ.

Die Rede von den früh industrialisierten kapitalistischen Gesellschaften des euroatlantischen Raums als Externalisierungsgesellschaften verweist auf das analytisch zentrale Faktum der uneingestandenen Voraussetzungen, Begleiterscheinungen und Folgewirkungen ihres welthistorischen Erfolgs. Eben diese, in der Regel unerzählte Geschichte des westlichen Industriekapitalismus aber ist dazu geeignet, auch dessen makroinstitutionelle Korrelate - die als koevolutionäre zivilisatorische Errungenschaften geltenden Institutionenordnungen der repräsentativen Demokratie und des demokratischen Wohlfahrtsstaats - in einem anderen, durchaus trüberen Licht erscheinen zu lassen. Die Externalisierungsperspektive nämlich lässt zutage treten, dass die den industriellen Wohlfahrtskapitalismus tragenden und für die sozialdemokratische Basiserzählung zentralen sozialen Kompromissstrukturen systematisch Kompromisse zu Lasten Dritter produziert haben. Als das - oder zumindest ein ganz entscheidendes - Geheimnis des wirtschaftlichen Erfolgs und des sozialen Zusammenhalts wohlfahrtskapitalistischer Demokratien muss daher der wahlweise intendierte oder implizierte, in jedem Fall aber objektiv funktionsnotwendige und subjektiv in Kauf genommene Misserfolg all jener gesehen werden, die von diesen Kompromissstrukturen ausgeschlossen bleiben.

Es ist hier nicht der Ort, um die Begriffsarchitektur des Externalisierungskonzepts detailliert darzulegen. Anhand von sieben Dimensionierungen des Begriffs soll jedoch wenigstens angedeutet werden, um welche Art von Strukturmechanismus es im Kern geht. Dabei gilt es zu betonen, dass funktionale Mechanismen nicht als solche gesellschaftlich wirksam werden, sondern nur vermittelt über die soziale Praxis real existierender Akteure, weswegen die nachfolgenden Kategorien jeweils auch 
spezifische - und in ihrer Kombination einen spezifischen Funktionszusammenhang konstituierende - Sozialpraktiken benennen.

Gleichsam der Urmechanismus externalisierender Vergesellschaftung ist jener der herrschaftlichen Aneignung produktionsrelevanter Güter und Ressourcen. Dabei geht es in erster Linie um die Aneignung von lebendiger Arbeit und lebendiger Natur - ein Prozess der Einverleibung von Wert, der für die Initiierung und Perpetuierung einer kapitalistischen Akkumulations- und Verwertungsdynamik unabdingbar ist. Von seinen historischen Anfängen an waren die materiellen Aneignungsanstrengungen des europäischen Kapitalismus im weltweiten Maßstab angelegt, und von Beginn an ging die Aneignung von Arbeit und Natur mit der Enteignung jener einher, die zuvor Herren ihrer selbst bzw. Nutznießende ihrer natürlichen Umwelt gewesen waren. An diesen Mechanismus schließt unmittelbar ein zweiter an, nämlich die wirtschaftliche Ausbeutung der zuvor angeeigneten Güter und Ressourcen. Ausbeutung soll hier in allgemeinster Weise als eine soziale Beziehung verstanden werden, in der es der machtvolleren Seite möglich ist, die sozial verletzbare Position der machtunterlegenen Gegenseite systematisch - und also immer wieder von neuem - zu ihrem einseitigen Vorteil auszunutzen (vgl. Tilly 1998, S. 117 ff.; Haubner 2017). In der Geschichte des europäischen Kapitalismus ist es den Zentrumsökonomien gelungen, an ihren Peripherien entsprechende Positionen der Verletzbarkeit und damit Ausbeutbarkeit, wo nicht ohnehin schon a priori gegeben, selbst aktiv herzustellen: zunächst in der klassischen, gewaltsamen Variante der Etablierung von kolonialen Herrschaftsbeziehungen und auf unfreier Arbeit beruhenden extraktivistischen Ökonomien, sodann in der moderneren, rechtsförmigen Gestalt des ungleichen ökonomischen und ökologischen Tauschs im Rahmen asymmetrischer globaler Arbeits-, Produktions- und Handelsregime (vgl. Boatcă 2015, S. 117 ff.). Der dritte Mechanismus, der die beiden zuvor genannten komplettiert bzw. überhaupt erst ermöglicht, ist jener der materiellen und symbolischen Abwertung all der Güter und Ressourcen, die nach ihrer Aneignung ausgebeutet werden sollen. Arbeit und Natur in anderen, als extern definierten gesellschaftlichen Räumen sind den industriekapitalistischen Externalisierungsgesellschaften zwar einerseits lieb, weil unmittelbar akkumulationsrelevant, andererseits aber gerade nicht teuer, da sie einem systematischen Prozess der Abwertung - bis hin zur Entwertung - unterliegen. Natur gilt dementsprechend als im Überfluss vorhanden und in niemandes Besitz sich befindend (bis man selbst den Eigentumstitel darauf erwirbt), sodass sie hemmungslos anzueignen und auszubeuten ist. Dasselbe gilt für die Arbeitskraft von „Wilden“ und Indigenen, Sklav*innen und Tagelöhner*innen, Frauen und Migrant*innen: In der Logik der Externalisierung sind auch sie und ihr Arbeitsvermögen preiswert zu haben, sie stellen die industriellen und reproduktiven Reservearmeen, deren wertschöpfende Quellen nie versiegen und die unkontrolliert gebraucht und nach Gutdünken vernutzt, also gleichsam entwertend verwertet werden können (vgl. Biesecker et al. 2013). Sie alle - die ,frei verfügbare“ Natur, das „schwächere“ Geschlecht, die „unterentwickelten“ Ökonomien - sind gesellschaftlich als das Andere der industriekapitalistischen Moderne markiert, das dem souveränen (nur am Rande: männlichweißen) Zugriff der mächtigeren Seite der jeweiligen Sozialbeziehung jederzeit und unbegrenzt offensteht. 
Wie kann nun dieser Funktionszusammenhang von abwertender Aneignung und Ausbeutung akkumulationsnotwendiger Güter und Ressourcen auf Dauer gestellt, auf stabil-dynamische Weise reproduziert werden? Die Antwort auf diese Frage gibt ein Set von vier weiteren Mechanismen. Zunächst wäre da die vierte im Bunde der Externalisierungsdimensionen, die Auslagerung selbst. Damit ist Externalisierung im engeren und eigentlichen Sinne angesprochen: Das intensive Bemühen, die Kollateralschäden und Folgekosten kapitalistischer Ausbeutungsbeziehungen so weit wie möglich in den externen Wirtschafts- und Sozialräumen zu belassen bzw. diese dorthin zu transferieren. Das entsprechende Kostenportfolio geht dabei über die strikt ökonomische Kostendimension - etwa in Form einer einseitig weltmarktabhängigen und damit extrem krisenanfälligen Spezialisierung peripherer Nationalökonomien weit hinaus. Auch die ökologischen Kosten der ohne Rücksicht auf Verluste betriebenen Rohstoff- und Energieproduktion, die sozialen Kosten der auf systematischer Überausbeutung von Arbeitskraft beruhenden Konsumgüter- und Dienstleistungsindustrien, die politischen und rechtlichen Kosten des häufig halblegalen oder kriminellen Agrar- und Fossilkapitalismus in den Ländern des globalen Südens sind immens und in jedem Sinne unkalkulierbar - berechenbar ist allein, dass sie eben nicht von den Externalisierungsgesellschaften selbst getragen werden müssen (vgl. I.L.A. Kollektiv 2017). Dafür suchen letztere auch mithilfe des fünften hier zu nennenden Mechanismus Sorge zu tragen: der möglichst effektiven bzw. kalkuliert selektiven Abschließung des eigenen Wirtschafts- und Sozialraums gegen das als solches konstruierte Außen. Vermochte das westlich dominierte Freihandelsregime für lange Zeit wirksam die wettbewerbsfähigen bzw. standardsetzenden industriellen Ökonomien des Zentrums zu protegieren, so sind die Migrationsregime der reichen Demokratien darauf angelegt, unkontrollierte Zuwanderung aus der restlichen Welt zu verhindern bzw. eine eigeninteressierte Arbeitskraftanwerbung zu ermöglichen. Auf beiden Wegen werden Schließungen im Sinne ökonomischer Chancenmonopolisierung vorgenommen - und damit Produktions- und Konsum-, Mobilitäts- und Lebenschancen für die Wirtschafts- und Sozialräume an den Peripherien systematisch reduziert. Die Funktionsfähigkeit des gesamten Externalisierungsgeschehens im Sinne seiner politischen Legitimation und sozialen Akzeptanz schließlich beruht auf zwei weiteren Mechanismen, nämlich zum einen auf der konsequenten Ausblendung des gesamten Praxiszusammenhangs von Aneignung, Ausbeutung, Abwertung, Auslagerung und Abschließung aus dem gesellschaftlich wirkmächtigen Wissenshaushalt sowie, zum anderen, auf der Aufschiebung der für die Externalisierungsgesellschaften selbst spürbaren Folgen des Externalisierungsgeschehens in eine vermeintlich entfernte Zukunft. In gewisser Weise stellen beide - Ausblendung und Aufschiebung - Externalisierungsmechanismen zweiter Ordnung dar, werden doch das Wissen um und die Erfahrbarkeit von Externalisierung selbst externalisiert. Einerseits wird das Wissen um die ,imperiale Lebensweise“ (Brand und Wissen 2017) der reichen Industrienationen vom gesellschaftlichen Kollektivbewusstsein abgespalten, auf beredte Weise kommunikativ beschwiegen oder aber in die Obhut der Bearbeitung durch spezialisierte Akteurssysteme (Wissenschaft, Kirchen, entwicklungspolitische NGOs, ehrenamtlich Engagierte) gegeben, um es dort auf für die Externalisierungslogik selbst folgenlose Weise prozessieren zu lassen. Andererseits werden die materialen Konsequenzen der fortgesetzten Externalisierungspolitik - 
nicht zuletzt auch für die Bevölkerungen der Externalisierungsgesellschaften selbst - systematisch in die Zukunft verlagert bzw. zu verlagern versucht, was die soziale Akzeptanz einer ,nachhaltigen Nicht-Nachhaltigkeit“ (Blühdorn 2018; Blühdorn et al. 2020) des Produzierens und Konsumierens entscheidend befördert, wenn nicht überhaupt erst ermöglicht.

Um die im Kern politökonomische Konstellation der sieben A's der Externalisierung herum, bzw. durch diese hindurch, konstituiert und etabliert sich historisch eine spezifische Form der Sozialität, die erst den Begriff der Externalisierungsgesellschaft rechtfertigt - als ein komplexes Ensemble sich wechselseitig stützender sozialer Praktiken, Subjektivitätsformen und Normativitäten (vgl. Lessenich 2018a). Dazu zählen einerseits die Selbstverständlichkeiten der alltäglichen Lebensführung großer Bevölkerungsmehrheiten in den Externalisierungsgesellschaften dieser Welt - von der praktisch grenzenlosen räumlichen Mobilität über die nicht enden wollenden Konsumangebote bis hin zu der Verfügbarkeit einer funktionsfähigen Infrastruktur öffentlicher Dienste und Einrichtungen. Andererseits materialisiert sich die politökonomische Externalisierungslogik in den Selbstverständnissen insbesondere der gesellschaftlich tonangebenden Mittelschichten hinsichtlich einer standesgemäßen Lebensführung und statuskonformer persönlicher Lebensentwürfe: Die Motivstrukturen und Handlungsorientierungen der Normalbürger*innen im westlichen Wohlfahrtskapitalismus sind auf das Engste mit bürgerlich-besitzindividualistischen Normen des materiellen Wohlstands, des sozialen Aufstiegs, der persönlichen Selbstverwirklichung und der individuellen wie kollektiven Potenzialnutzung verbunden (vgl. Lessenich 2018b). Diese sozialen Selbstverständnisse und Selbstverständlichkeiten sind wiederum eingebettet in einen permanenten Prozess expliziter wie insbesondere auch impliziter gesellschaftlicher Selbstverständigung über die Angemessenheit und Legitimität jener Weltverhältnisse, in denen sich das eigene Leben abspielt. Die Externalisierungsgesellschaft zehrt von einer wirtschaftsliberalen Moralökonomie, die das eigene ökonomische Gebaren als ethisch neutral ausweist: Märkte und „,marktgerechte" Ergebnisse entziehen sich demnach moralischen Bewertungen, Freihandel gilt als ein die komparativen Vorteile aller Beteiligten zur Geltung bringendes Positivsummenspiel und die Erde zwar nicht als eine Scheibe, wohl aber die globale Ökonomie als eine einzige Wettbewerbsplattform mit systemischen Aufholchancen für all jene Nachzügler, die sich in ihre Weltmarktnischen begeben und dort zu bewähren wissen.

All dies verweist auf die tiefgreifenden sozialisierenden Effekte der Externalisierungsgesellschaft: In ihren individuellen Handlungsorientierungen und sozialen Deutungsmustern auf das engste mit deren Funktionsmechanismen verstrickt, sind die Bürger*innen der Externalisierungsgesellschaft Teil eines institutionellen Arrangements, dem sie nicht qua individueller Willensentscheidung entkommen können - und an das sie zugleich ganz basale materielle wie ideelle Interessen binden. Insofern leben sie in einer Art erzwungener Komplizenschaft, im Zustand teilnehmender Zwangsintegration: Ihre gewohnten Lebensverhältnisse, die eingeübten Alltagspraktiken, der erworbene Lebensstandard, die erreichten Berechtigungsniveaus lassen sich über die Zeit nur durch die permanente Reproduktion der Externalisierungslogik aufrechterhalten. Die Bürger*innen der Externalisierungsgesellschaft sind also abhängig von deren fortgesetzter Funktionsfähigkeit - und in diesem spezifischen 
Sinne sind sie Beherrschte und Herrschende zugleich: Ohne auch nur ansatzweise über die Entscheidungsgewalt über die historisch-konkrete Form ihrer Vergesellschaftung zu verfügen, in der nationalgesellschaftlichen Verteilungsstruktur von Lebenschancen im Zweifel auf einer der untersten Stufen situiert, haben sie doch zugleich materiell und symbolisch immer auch an den Externalisierungsdividenden teil, die durch die gesellschaftssystemische Praxis der Aneignung, Abwertung und Ausbeutung, der Auslagerung, Abschließung, Ausblendung und Aufschiebung entstehen. Mitgehangen, mitgefangen: Es gibt kein richtiges Leben in der Externalisierungsgesellschaft.

\section{Grenzen der Solidarität oder: Die Doppelmoral von der Geschicht'}

Diese Einsicht aber wiederum verweist auf die Möglichkeit einer immanenten Kritik der Externalisierung bzw., genauer, einer reflexiven Kritik der Beherrschten an ihrer erzwungenen Herrschaft: Die Bürger*innen der Externalisierungsgesellschaft können faktisch ihre jeweilige Position im System sozialer Ungleichheit nur halten und ihre wie auch immer geartete Teilhabe an dem über öffentliche Institutionen der Wohlfahrtsproduktion etablierten Zusammenhang wechselseitiger Anerkennung sozialer Sicherheits- und Schutzansprüche nur realisieren, indem sie Dritte, außerhalb dieses Zusammenhangs Stehende, systematisch schädigen. Die Funktionsweise der Externalisierungsgesellschaft basiert gewissermaßen auf der Umkehrung des Rawls'schen Differenzprinzips: Selbst die am schlechtesten Gestellten können ihre soziale Lage nur auf Kosten anderer sichern, in der Logik der Externalisierung geht jede absolute oder relative Verbesserung ihrer Lage mit der Erhöhung dieser Kosten einher.

Erst auf dieser Basis wird in der Externalisierungsgesellschaft überhaupt „Solidarität“" möglich, lassen sich Programme des Bedarfs- und Risikoausgleichs, die Produktion von öffentlichen Gütern oder die Gewährleistung sozialer Hilfen organisieren und finanzieren. Solidarität in der Externalisierungsgesellschaft ist mithin - jenseits spezifischer, weitergehender Praktiken des propagierten oder realisierten Ausschlusses aus der nationalen Solidargemeinschaft (vgl. Brosch 2007; Dörre 2016) - grundsätzlich exklusive bzw. exkludierende Solidarität. Selbst wenn man etwa alle Kritik an der geltenden Variante der Grundsicherung für Arbeitsuchende - eine der letzten Rückfallpositionen individueller Existenzsicherung im deutschen Wohlfahrtsstaat - außer Acht lässt und diese als einen Akt institutionalisierter sozialer Solidarität mit den Schwächsten, sprich den in einer durch und durch auf Erwerbsarbeit gepolten Gesellschaft erwerbslos Bleibenden, versteht, so steht dahinter doch eine bittere Wahrheit: Nur dank der ,künstlichen“, faktisch aber äußerst gewaltsamen Absenkung der sozialen Reproduktionskosten der Arbeitskraft weil man eben hierzulande Kleidung und Nahrungsmittel, Energie und elektronische Geräte zu externalisierungsbedingten Spottpreisen kaufen kann -, lässt sich in Deutschland von einem Haushaltseinkommen auf Hartz IV-Niveau überhaupt leben bzw. damit überleben. Und sogar noch diese sozial überaus voraussetzungsvolle und fremdschädigende, für sich selbst genommen aber zugleich äußerst dürftige und selbstachtungsgefährdende Form des sozialpolitisch organisierten sozialen Zusam- 
menhalts wird bekanntlich im öffentlichen Diskurs immer mal wieder als ,zu teuer“, als „sozialer Fehlanreiz“ oder als Magnet für die „Einwanderung in die Sozialsysteme" qualifiziert - woraufhin in der Regel weitergehende Maßnahmen zur Sicherung der sozialen Exklusivität des Sicherungssystems ergriffen werden, sei es in Form von gruppenbezogenen Sanktionsregelungen oder der Absenkung von Leistungen unter den vermeintlichen „Grundsicherungs“-Standard.

Externalisierungsgesellschaftlich gesehen hat der demokratisch-kapitalistische Wohlfahrtsstaat also noch ein zweites Gesicht: Im globalen Zusammenhang betrachtet ist er eine große Entsolidarisierungs- bzw. Solidaritätsbegrenzungsmaschinerie. Und zwar grundsätzlich, schon von der Logik seiner Konstitution her, also auch ganz ohne seine jüngere „,neoliberale“ Sub- bzw. Perversion. Begreift man die „Idee eines wechselseitigen Zusammenhangs zwischen den Mitgliedern einer Gruppe von Menschen“ (Bayertz 1998, S. 11) als den deskriptiven Kern des Solidaritätskonzepts und die Vorstellung von ,wechselseitigen Bindungen und Verpflichtungen“ (ebd., S. 49) zwischen diesen Gruppenmitgliedern als eine breit akzeptanzfähige, weil relativ anspruchslose normative Aufladung desselben, so lassen sich mit dem analytischen Instrumentarium der Externalisierungsdiagnose durchaus vielfältige wechselseitige Zusammenhänge zwischen den Bürger*innen wohlfahrtskapitalistischer Demokratien im euroatlantischen Raum und ihrer globalen sozialen Umwelt beschreiben. Doch sind diese transnationalen Zusammenhänge historisch in keiner Weise zum Bezugspunkt der Institutionalisierung wechselseitiger Bindungen und Verpflichtungen in den demokratisch-kapitalistischen Wohlfahrtsstaaten der westlichen Welt geworden: Deren materiale Bindungswirkung bleibt bis heute ganz der Ideenwelt nationaler Solidarität verpflichtet.

Die Grenzziehungen, die dieser historisch-konkreten Solidaritätskonzeption inhärent sind, lassen sich wiederum, mit der neomarxistischen Relektüre Max Webers durch die Soziologen Frank Parkin (1983) und Raymond Murphy (1984), als multiple Strukturdynamiken sozialer Schließung verstehen. Nach innen nehmen die industriekapitalistischen Gesellschaften im Prozess ihrer Verwohlfahrtsstaatlichung eine zweifache Schließung des Raums institutionalisierter Solidarität vor. Eine erste Grenzziehung - unterhalb jener vorgelagerten, kapitalismuskonstitutiven Metagrenze zwischen Produktionsmitteleigentümern und Arbeitskraftbesitzern - ist die des industriekapitalistischen Geschlechterverhältnisses: „Die Zuweisung zu den Geschlechtscharakteren ist Basis der Industriegesellschaft" (Beck 1986, S. 174), diese gründet ,auf einer unvollständigen, genauer: halbierten Vermarktung menschlichen Arbeitsvermögens“ (ebd., Hervorhebungen i. Orig.). Die ,geschlechtsständischen Zuweisungen“" (Beck 1986, S. 177) des Industriekapitalismus spiegeln sich in einer modernen, zuallererst geschlechtersegregierten „Ständehierarchie“ (ebd.) des Solidaritätsverständnisses: Während die männlichen Produktionsarbeiter sich sozialpolitisch ihrer wechselseitigen Unterstützung in den Wechselfällen der Lohnabhängigenexistenz versichern, werden die weiblichen Reproduktionsarbeiterinnen in den Stand der Lohnabhängigenabhängigkeit relegiert und auf von den Solidaritätsansprüchen ihrer Ehemänner abgeleitete Sicherungsansprüche verwiesen. Eine zweite, komplementäre innere Grenzziehung ist die zwischen den Einheimischen und den Zugewanderten - eine klassische Etablierten-Außenseiter-Figuration (vgl. Elias und Scotson 1990), an die im Industriekapitalismus eine arbeitsmarktstrukturelle Spal- 
tung bzw. eine „horizontale Disparität“ (vgl. Borchert und Lessenich 2016, S. 49 ff.) zwischen unterschiedlichen Gruppen von Produktionsarbeiter*innen anschließt. Die Etablierung eines Gastarbeiterregimes in den westeuropäischen Industriegesellschaften nach dem Zweiten Weltkrieg führte zur systematischen „Unterschichtung“ der nationalen Beschäftigungssysteme und äußerte sich nicht nur in symbolischen Abgrenzungen zwischen den Angehörigen ethnisch markierter Arbeitsmarktsegmente, sondern auch in einer materiellen Strukturierung des Systems sozialer Rechtsansprüche und Solidaritätsverbürgungen entlang der rechtlich-politischen Statusdifferenzen unter den erwerbstätigen Nicht-Staatsbürger*innen (vgl. für Deutschland Karakayali 2008).

Genau hier liegt denn auch die Schnittstelle zu den Grenzziehungen industriekapitalistischer Solidaritätsarrangements nach außen. Paradoxerweise nämlich (oder gerade auch nicht) stellt die Sozialkategorie der „citizenship“ bzw. des Staatsbürger*innenstatus, in der soziologischen Theorietradition Thomas H. Marshalls seit jeher als Instanz der Inklusion tendenziell der gesamten Bevölkerung in tendenziell sämtliche Funktions- und Leistungssysteme der demokratisch-industriekapitalistischen Gesellschaft gewürdigt (vgl. Marshall 1992; Mackert 1999), gleichursprünglich auch ein Instrument der effektiven sozialen Exklusion dar. „Citizenship laws in industrialized capitalist countries ... operate to prevent the dilution of the benefits of industrialization (spread them more thinly among a large number) through the exclusion of people born elsewhere" (Murphy 1984, S. 559; vgl. Scherschel 2018). Neben „,class“, „gender“ und „race“ als Strukturkategorien solidaritätspolitischer Grenzziehungen kommt damit eine weitere, vierte ins Spiel: die Strukturkategorie „place“ (vgl. Milanovic 2012), auf deren Grundlage (bzw. im Wortsinne: auf deren Boden) die drei genannten sozialen Strukturierungsformen operieren. Der Geburtsort einer Person und die ihr damit zukommende Staatsan- und -zugehörigkeit ist die allererste, fundamentale Grenze der Solidarität, die von nationalen Wohlfahrtsstaaten gezogen und institutionalisiert wird. Diese Grenzziehung impliziert, über die geschlechter- und migrationspolitische Halbierung industriekapitalistischer Solidaritätsbeziehungen hinaus, ,an additional line of class cleavage beneath property classes, namely, the line separating the working class of advanced capitalist countries from the poor of the Third World" (Murphy 1984, S. 559). Staatsbürger*innenrechte, ihre institutionelle Gewährleistung bzw. die Negation eben dieser Gewährleistung, ziehen eine den wohlfahrtsstaatsinternen Berechtigungsstrukturen vorgängige Solidaritätsgrenze, durch die sich die nationale Solidargemeinschaft, ,including the most disadvantaged fraction of the working class“ (ebd.), vor etwaigen Solidaritätsansprüchen aus ihrer sozialen Außenwelt schützt. Raymond Murphy bezeichnet die außerhalb der industriekapitalistischen Zentren lebenden Arbeiterklassen daher als eine ,citizenship underclass“ (ebd.), die von den inneren Prozessen demokratischkapitalistischer Statuszuweisung ausgeschlossen, in ihren Lebenschancen aber direkt oder indirekt von den Auswirkungen dieser Prozesse betroffen ist.

Historisch-soziologisch lassen sich diese Zusammenhänge in die Figur eines doppelten Gesellschaftsvertrags übersetzen, der die demokratischen Kapitalismen des Westens und deren wohlfahrtsstaatliche Entwicklung im 20. Jahrhundert, insbesondere über die lange Nachkriegszeit hinweg, getragen hat. Im Kern bestand der westliche Gesellschaftsvertrag aus einem sozialen Tauschverhältnis: Die politische 
Akzeptanz von Privateigentum und Unternehmerfreiheit wurde im Austausch gegen die wachsende soziale Teilhabe der nicht-besitzenden Klassen am Wohlstand der jeweiligen Nation sichergestellt. Seinen institutionellen Ausdruck fand dieses Arrangement im industriellen Solidaritätspakt des modernen Wohlfahrtsstaats, seinen kulturellen Niederschlag in der Demokratisierung und damit einhergehenden Ausweitung von Konsumchancen. In unterschiedlichen Varianten und historisch phasenverschoben ist dieser ,implizite Vertrag“ (vgl. Moore 1982, S. $38 \mathrm{ff}$.) zu einem basalen Bestandteil des gesellschaftlichen Selbstverständnisses geworden - semantisch repräsentiert etwa in der Formel von der „Sozialen Marktwirtschaft“ in der Bundesrepublik oder vom „New Deal“ in den Vereinigten Staaten.

Doch der demokratisch-kapitalistische Gesellschaftsvertrag hatte noch eine zweite Seite. Er beruhte nämlich seinerseits auf der klassenübergreifenden Übereinkunft, dass die negativen Konsequenzen, die der industrielle Wohlfahrtskapitalismus als Wachstums- und Verteilungsmodell außerhalb der eigenen Grenzen zeitigte, von den euroatlantischen Nationen selbst effektiv fernzuhalten seien. Die Ausbeutung natürlicher Ressourcen und die Überausbeutung von Arbeitskraft in entfernten Regionen jenseits der westlichen Welt, ohne die deren Produktions- und Konsumweisen gar nicht möglich und aufrechtzuerhalten gewesen wären, sollten möglichst reibungslos und unbemerkt erfolgen. Diese Kehrseite der vielgerühmten Erfolgsgeschichten des (mehr oder weniger) ,sozialen Kapitalismus“ wurde demensprechend nicht - weder in Deutschland noch in den USA oder einem anderen westlichen Wohlfahrtsstaat an die große Glocke gehängt. Sie war das Kleingedruckte, das eigentlich Implizite des impliziten Gesellschaftsvertrags.

Heute aber ist dieser doppelte Gesellschaftsvertrag prekär geworden, und zwar in seinen beiden Dimensionen. Dem immer noch wachsenden Reichtum der industriekapitalistischen Nationen zum Trotz sind soziale Sicherheitsgarantien und Aufstiegsversprechen bis in die Mitte der Gesellschaft hinein erschüttert worden, die Erfahrung prekärer Arbeits- und Lebensbedingungen stellt keineswegs nur noch ein soziales Randphänomen dar. Die Anschlussfähigkeit soziologischer Diagnosen einer sich ankündigenden „Abstiegsgesellschaft“ (Nachtwey 2016) in der hiesigen Öffentlichkeit spricht ebenso für eine tiefgreifende Verunsicherung breiter Bevölkerungsschichten wie der unvermittelte politische Erfolg Donald Trumps bei der amerikanischen Präsidentschaftswahl. Wie der Aufstieg neonationalistischer Bewegungen in Deutschland und Europa verweist der „Trump-Effekt“ zudem auf die Tatsache, dass für die wahrgenommene Erosion des demokratisch-kapitalistischen Gesellschaftsvertrags nicht „die Wirtschaft“ bzw. die ökonomischen Funktionseliten verantwortlich gemacht werden, sondern vielmehr ,die Politik“ bzw. die ,politische Klasse“ (vgl. Borchert und Lessenich 2016, S. 123 ff.).

Die politisch Herrschenden geraten nun aber zugleich von einer zweiten Seite unter Druck. Denn auch die Geheimklausel des demokratisch-kapitalistischen Kompromisses wird zunehmend unwirksam. Auch in dieser Hinsicht machen die westlichen Gesellschaften gegenwärtig die Erfahrung, dass die Bedingungen ihrer stabilen Reproduktion brüchig werden. Klimawandel und Migration sind die Chiffren für die ins öffentliche Bewusstsein rückende Brüchigkeit jener beiden Fundamente, auf denen das gesamte euroatlantische Gesellschaftsmodell ruht: das fossilistische Energieregime und die globale Ungleichheitsordnung. Es ist daher nicht allein die 
im engeren Sinne sozioökonomische Lage breiter Bevölkerungsmehrheiten, aus der sich ein gesellschaftliches Gefühl der Prekarität - im Wortsinne: der Widerrufbarkeit eines einmal erreichten Zustands oder Statuses - speist. Das Unbehagen im und am demokratischen Wohlfahrtskapitalismus reicht tiefer: Es gründet in der unheimlichen Ahnung einer in jeder Hinsicht unhaltbaren Lebensweise, die sich eben nicht länger auf die Unendlichkeit natürlicher Ressourcen und das Stillhalten des Drittweltproletariats verlassen kann.

Damit aber wird der fundamentale Widerspruch offenkundig, in dem sich die Bürger*innen der Externalisierungsgesellschaft in ihrer übergroßen Mehrzahl strukturbedingt bewegen, mithin zwangsläufig bewegen müssen: Selbst von den zentralen Machtressourcen - dem Besitz von Produktivvermögen - ausgeschlossen, sind sie in ihrer sozialen Existenz nicht nur von den sie ausschließenden Machthabenden abhängig, sondern zugleich davon, dass andere - strukturell noch machtlosere - Gruppen aus dem demokratisch-kapitalistischen Verteilungsspiel ausgeschlossen werden. Mit Murphy (1984, S. 562) lässt sich hier von einer geradezu schizophrenen Positionierung sprechen, von einer ,Jekyll and Hyde relationship“, in der die ,derivative exclusionary groups“ - die Beherrschten (in) der Externalisierungsgesellschaft - zu der ,principal exclusionary group“ - den sie selbst Beherrschenden - stehen: ,the former are dependent on and hence allied with the latter and yet they are dominated and excluded by the latter and therefore provoked to usurp the latter's exclusive power and advantages".

Wie kann, wie wird dieses Spannungsverhältnis, den intern Herrschenden mehr Wohlfahrt abtrotzen zu wollen, damit aber unweigerlich die Wohlfahrt der extern Beherrschten in Mitleidenschaft ziehen zu müssen, aufgelöst werden? Eine Möglichkeit, die nicht nur denkbar ist, sondern für die es gegenwärtig unverkennbare Anzeichen gibt, ist eine Anpassung der wohlfahrtsstaatlichen Basiserzählung im Sinne einer offensiveren, aggressiveren Betonung des exklusiven Charakters des sozialpolitisch organisierten gesellschaftlichen Zusammenhalts - des Nationalen am Sozialen also. Dies wäre bzw. ist gleichbedeutend mit der zunächst diskursiven, in der Folge aber auch materialen Forcierung der externalisierungstypischen solidaritätspolitischen Doppelmoral, wobei diese Strategie durchaus milieuspezifisch variiert (vgl. Koppetsch 2018): von den naturalisierenden Festschreibungen sozialer Hierarchien in den Oberschichten über die Versuche zur Wiederherstellung des kulturellen Alleinvertretungsanspruchs der (männlich-weißen) Mittelklassen bis hin zur symbolischen Abwertung von Außenseitern im Rahmen der sozialen Verteilungskonflikte am unteren Ende der Sozialstruktur. In jedem Fall aber werden hier die Solidaritätsgrenzen - gegenüber Unterklassen, Frauen, Zugewanderten, Ferngehaltenen - verschärft, werden andere als die eigenen Emanzipationsansprüche und -anliegen abgewiesen.

Eine zweite, entgegensetzte Möglichkeit, die einstweilen gesellschaftlich deutlich weniger prominent verhandelt wird, wären veränderte Praktiken der Solidarität (vgl. Lessenich 2019, S. 96ff.). Es wären Praktiken, die das Solidaritätsarrangement des demokratisch-industriekapitalistischen Wohlfahrtsstaats nicht - nach dem „,neoliberalen“ Angriff - zu rekonstituieren, sondern vielmehr zu transzendieren suchen. Solidarische Praktiken, denen eine neue wohlfahrtsstaatliche Basiserzählung zugrunde läge. Und zwar die Erzählung von Solidarität als einer Praxis, die ko- 
operativ, performativ und transformativ in einem ist: kooperativ, indem die Idee wechselseitiger Verbundenheit nicht im Sinne eines stellvertretenden Einstehens für die Belange anderer, sondern des gemeinsamen Eintretens für beiderseitig geteilte Belange ausbuchstabiert wird; performativ, indem nicht die Vorstellung einer vorgängigen solidarischen Haltung bedient wird, der bei Bedarf nur noch individuell Ausdruck zu geben wäre, sondern vielmehr klargestellt wird, dass das Bewusstsein wechselseitiger Verbundenheit nur im Akt des gemeinsamen Eintretens für geteilte Belange wächst (die sich wiederum als solche erst über den „Umweg“ dieses kollektiven Handelns erweisen); und transformativ, indem als Ziel der aus wechselseitiger Verbundenheit resultierenden Verpflichtungen nicht etwa die Aufrechterhaltung oder Wiederherstellung einer gegebenen Privilegienstruktur ausgegeben wird, sondern die grundlegende Veränderung des gesellschaftlichen Systems ungleicher Lebenschancen.

Insofern wird die Geschichte des westlichen Wohlfahrtskapitalismus in doppelter Weise umgeschrieben werden müssen. Retrospektiv durch historische Sozio$\log *$ innen, die systematisch die weltgesellschaftlichen Voraussetzungen, Begleiterscheinungen und Folgewirkungen nationalgesellschaftlich institutionalisierter Solidarität rekonstruieren. Prospektiv aber durch gesellschaftliche Individuen, deren soziale Praxis geeignet ist, Solidarität über die Grenzen und Begrenzungen ihrer bisherigen Institutionalisierung im nationalen Wohlfahrtsstaat hinauszutreiben.

Funding Open Access funding provided by Projekt DEAL.

Open Access Dieser Artikel wird unter der Creative Commons Namensnennung 4.0 International Lizenz veröffentlicht, welche die Nutzung, Vervielfältigung, Bearbeitung, Verbreitung und Wiedergabe in jeglichem Medium und Format erlaubt, sofern Sie den/die ursprünglichen Autor(en) und die Quelle ordnungsgemäß nennen, einen Link zur Creative Commons Lizenz beifügen und angeben, ob Änderungen vorgenommen wurden.

Die in diesem Artikel enthaltenen Bilder und sonstiges Drittmaterial unterliegen ebenfalls der genannten Creative Commons Lizenz, sofern sich aus der Abbildungslegende nichts anderes ergibt. Sofern das betreffende Material nicht unter der genannten Creative Commons Lizenz steht und die betreffende Handlung nicht nach gesetzlichen Vorschriften erlaubt ist, ist für die oben aufgeführten Weiterverwendungen des Materials die Einwilligung des jeweiligen Rechteinhabers einzuholen.

Weitere Details zur Lizenz entnehmen Sie bitte der Lizenzinformation auf http://creativecommons.org/ licenses/by/4.0/deed.de.

\section{Literatur}

Arrighi, G. (1994). The long twentieth century. Money, power, and the origins of our times. London: Verso. Bayertz, K. (1998). Begriff und Problem der Solidarität. In K. Bayertz (Hrsg.), Solidarität. Begriff und Problem (S. 11-53). Frankfurt a. M.: Suhrkamp.

Beck, U. (1986). Risikogesellschaft. Auf dem Weg in eine andere Moderne. Frankfurt a. M.: Suhrkamp.

Biesecker, A., Hofmeister, S., \& Winterfeld, U. v. (2013). Draußen? Zur Dialektik von Aneignung und Enteignung und zu deren aktuellen Erscheinungsformen. Das Argument, 55, 522-538.

Blühdorn, I. (2018). Nicht-Nachhaltigkeit auf der Suche nach ihrer politischen Form. Konturen der demokratischen Postwachstumsgesellschaft. Berliner Journal für Soziologie, 28, 151-180.

Blühdorn, I., Butzlaff, F., Deflorian, M., Hausknost, D., \& Mock, M. (2020). Nachhaltige Nicht-Nachhaltigkeit. Warum die ökologische Transformation der Gesellschaft nicht stattfindet. Bielefeld: transcript.

Boatcă, M. (2015). Global inequalities beyond occidentalism. Farnham: Ashgate. 
Borchert, J., \& Lessenich, S. (2016). Claus Offe and the critical theory of the capitalist state. New York: Routledge.

Brand, U., \& Wissen, M. (2017). Imperiale Lebensweise. Zur Ausbeutung von Mensch und Natur im globalen Kapitalismus. München: oekom.

Brosch, M. (2007). Exklusive Solidarität. Linker Antisemitismus in Deutschland: Vom Idealismus zur Antiglobalisierungsbewegung. Berlin: Metropol.

Brown, W. (2018). Die schleichende Revolution. Wie der Neoliberalismus die Demokratie zerstört. Berlin: Suhrkamp.

Butterwegge, C. (2015). Hartz IV und die Folgen. Auf dem Weg in eine andere Republik? Weinheim: Beltz Juventa.

Dörre, K. (2016). Furcht vor Veränderung. Abstiegsängste und exklusive Solidarität. In J. Klose und W. Schmitz (Hrsg.), Freiheit, Angst und Provokation. Zum gesellschaftlichen Zusammenhalt in der postdiktatorischen Gesellschaft (S. 331-336). Dresden: Thelem.

Dyk, S. v., \& Schauer, A. (2015). ,...daß die offizielle Soziologie versagt hat“. Zur Soziologie im Nationalsozialismus, der Geschichte ihrer Aufarbeitung und der Rolle der DGS. 2. Auflage. Wiesbaden: Springer VS.

Elias, N., \& Scotson, J. L. (1990). Etablierte und Außenseiter. Frankfurt a. M.: Suhrkamp.

Esping-Andersen, G. (1985). Politics against markets. The social democratic road to power. Princeton: Princeton University Press.

Esping-Andersen, G. (1990). The three worlds of welfare capitalism. Cambridge: Polity Press.

Esping-Andersen, G. (1999). Social foundations of postindustrial economies. Oxford: Oxford University Press.

Haubner, T. (2017). Ein unbequemes Erbe. Die Ausbeutung von Pflegearbeiten und der marxistische Ausbeutungsbegriff. Das Argument, 59, 534-547.

Herz, T. (1996). Die „Basiserzählung“ und die NS-Vergangenheit. Zur Veränderung der politischen Kultur in Deutschland. In L. Clausen (Hrsg.), Gesellschaften im Umbruch. Verhandlungen des 27. Kongresses der Deutschen Gesellschaft für Soziologie in Halle an der Saale 1995 (S. 91-109). Frankfurt a. M.: Campus.

I.L.A. Kollektiv (2017). Auf Kosten anderer? Wie die imperiale Lebensweise ein gutes Leben für alle verhindert. München: oekom.

Karakayali, S. (2008). Gespenster der Migration. Zur Genealogie illegaler Einwanderung in der Bundesrepublik Deutschland. Bielefeld: transcript.

Koppetsch, C. (2018). Rechtspopulismus als Klassenkampf? Soziale Deklassierung und politische Mobilisierung. WSI-Mitteilungen, 71, 382-391.

Korpi, W. (1983). The democratic class struggle. London: Routledge \& Kegan Paul.

Lessenich, S. (1999). Vorwärts - und nichts vergessen. Die neue deutsche Sozialstaatsdebatte und die Dialektik sozialpolitischer Intervention. PROKLA, 29, 411-430.

Lessenich, S. (2012). Theorien des Sozialstaats zur Einführung. Hamburg: Junius.

Lessenich, S. (2017). Rezension: Immer, nur und überall: Wendy Browns Kritik des Neoliberalismus. Ethik und Gesellschaft, 1/2017. https://doi.org/10.18156/eug-1-2017rez-3. Zugegriffen: März 2020.

Lessenich, S. (2018a). Externalisierung als soziale Praxis - Wachstum als Lebensform. Kommentar zum Hauptbeitrag von Rahel Jaeggi. Zeitschrift für Wirtschafts- und Unternehmensethik, 19, 423-428.

Lessenich, S. (2018b). Die ewige Mitte und das Gespenst der Abstiegsgesellschaft. In N. M. Schöneck \& S. Ritter (Hrsg.), Die Mitte als Kampfzone. Wertorientierungen und Abgrenzungspraktiken der Mittelschichten (S. 163-178). Bielefeld: transcript.

Lessenich, S. (2019). Grenzen der Demokratie. Teilhabe als Verteilungsproblem. Ditzingen: Reclam.

Mackert, J. (1999). Kampf um Zugehörigkeit. Nationale Staatsbürgerschaft als Modus sozialer Schließung. Wiesbaden: Westdeutscher Verlag.

Manow, P. (2002). „The Good, the Bad, and the Ugly“. Esping-Andersens Sozialstaatstypologie und die konfessionellen Wurzeln des westlichen Wohlfahrtsstaats. Kölner Zeitschrift für Soziologie und Sozialpsychologie, 54, 203-225.

Marshall, T. H. (1992) [1949]. Bürgerrechte und soziale Klassen. Zur Soziologie des Wohlfahrtsstaates. Frankfurt a. M.: Campus.

Marx, K. (1986) [1867]. Das Kapital. Kritik der politischen Ökonomie. Erster Band. MEW Band 23. Berlin: Dietz.

Milanovic, B. (2012). Global inequality: From class to location, from proletarians to migrants. Global Policy, 3, 125-134.

Moore, B. (1982). Ungerechtigkeit. Die sozialen Ursachen von Unterordnung und Widerstand. Frankfurt a. M.: Suhrkamp. 
Morris, L. (2002). Managing migration. Civic stratification and migrants' rights. London: Routledge.

Murphy, R. (1984). The structure of closure: A critique and development of the theories of Weber, Collins, and Parkin. The British Journal of Sociology, 35, 547-567.

Nachtwey, O. (2009). Marktsozialdemokratie. Die Transformation von SPD und Labour Party. Wiesbaden: VS Verlag für Sozialwissenschaften.

Nachtwey, O. (2016). Die Abstiegsgesellschaft. Über das Aufbegehren in der regressiven Moderne. Berlin: Suhrkamp.

Offe, C., \& Wiesenthal, H. (1985). Two logics of collective action. In C. Offe, Disorganized Capitalism (S. 170-220). Cambridge, MA: The MIT Press.

Orloff, A. S. (1993). Gender and the social rights of citizenship: The comparative analysis of gender relations and welfare states. American Sociological Review, 58, 303-328.

Parkin, F. (1983). Strategien sozialer Schließung und Klassenbildung. In R. Kreckel (Hrsg.), Soziale Ungleichheiten. Soziale Welt - Sonderband 2 (S. 121-135). Göttingen: Otto Schwartz.

Scherschel, K. (2018). An den Grenzen der Demokratie - Citizenship und Flucht. Berliner Journal für Soziologie, 28, 123-150.

Shalev, M. (1983). The social democratic model and beyond: Two „generations“ of comparative research on the welfare state. Comparative Social Research, 6, 315-351.

Streeck, W. (2013). Gekaufte Zeit. Die vertagte Krise des demokratischen Kapitalismus. Berlin: Suhrkamp. Tilly, C. (1998). Durable inequality. Berkeley: University of California Press.

Wallerstein, I. (2004). World-Systems Analysis. An introduction. Durham: Duke University Press.

Stephan Lessenich geb. 1965. Professor für Politische Soziologie sozialer Ungleichheit an der LudwigMaximilians-Universität München. Forschungsschwerpunkte: Politische Soziologie sozialer Ungleichheit, Vergleichende Wohlfahrtsstaatsforschung, Kapitalismusanalyse und -kritik, Soziologie des Alter(n)s. Ausgewählte Veröffentlichungen: (mit J. Borchert) Claus Offe and the critical theory of the capitalist state, 2016; Neben uns die Sintflut. Die Externalisierungsgesellschaft und ihr Preis, 2016; Grenzen der Demokratie, 2019. 